\title{
DNA polymerase $\theta$-a new target for synthetic lethality?
}

$\mathrm{M}$

any cancers harbour deficiencies

in DNA double-strand break

(DSB) repair; in particular, aberrations affecting components of the homologous recombination (HR) pathway, such as BRCA1 and BRCA2, and ATM, are common. However, alternate DSB-repair pathways, such as non-homologous endjoining (NHEJ), enable these cancers to persist. Agents that promote DSBs, and ultimately overwhelm these alternate repair mechanisms to cause lethal DNA damage, such as poly(ADP-ribose) polymerase inhibitors, have emerged as effective treatments for HR-deficient cancers-a therapeutic strategy termed 'synthetic lethality'. Now, two papers report a key role for the low-fidelity DNA polymerase $\theta$ $(\operatorname{Pol} \theta)$ in microhomology-mediated end joining (MMEJ), a PARP1-dependent, error-prone, alternative NHEJ pathway, and demonstrate that MMEJ is important for the survival of HR-deficient cancer cells. Thus, inhibition of $\mathrm{Pol} \theta$ in HR-deficient cancers might promote synthetic lethality.

In these papers, $\operatorname{Pol} \theta$ was identified as a crucial component of the MMEJ pathway by distinct approaches. Agnel Sfeir and co-workers were assessing differences in the sequence fidelity of the classic NHEJ and MMEJ repair mechanisms in mouse cells made vulnerable to telomere fusion. In the course of their experiments, knockdown of the gene encoding Pol $\theta$ was found to reduce the frequency of telomere fusions, and also chromosomal translocations, via abrogation of MMEJ, but not classic NHEJ.

By contrast, Alan D'Andrea and colleagues were investigating whether specialized DNA polymerases involved in the repair of DNA damage by a process called translesion synthesis are upregulated in cancer to mitigate the effects of other DNA-repair deficiencies. Their screen of polymerase gene-expression profiles in multiple cancers revealed that $\operatorname{Pol} \theta$ was consistently overexpressed specifically in epithelial ovarian cancers. Furthermore, upregulation of $\mathrm{Pol} \theta$ correlated with expression of many components of the HR pathway and with tumour grade. "Since ovarian cancers harboured a high frequency of alteration in the HR repair pathway, we hypothesized that $\mathrm{Pol} \theta$ expression could be triggered by an HR defect," explains D'Andrea. In fact, both groups went on to examine the relationship between Pol $\theta$-mediated MMEJ and HR repair.

"In parallel to promoting MMEJ, our data show that Pol $\theta$ can inhibit HR, thereby acting as a key factor in orchestrating DNA repair; on the one hand it promotes error-prone repair, while at the same time blocking error-free repair," says Sfeir. In their model, Sfeir et al. discovered that cells lacking Pol $\theta$ had an increased rate of $\mathrm{HR}$, as demonstrated by telomere sister chromatid exchange events, as well as an increased number of RAD51 foci, which are critical for HR. Interestingly, D’Andrea and co-workers also noted enhanced HR-mediated repair and increased numbers of RAD51 foci upon knockdown of $\operatorname{Pol} \theta$, and demonstrated that $\mathrm{Pol} \theta$ binds directly to RAD51; this interaction was necessary for inhibition of HR. These findings suggest that $\operatorname{Pol} \theta$ sequesters RAD51 and prevents formation of HR-repair complexes, which are toxic in BRCA1/2-deficient cells.

The studies also investigated the role of MMEJ in cells with deficient HR repair mechanisms. "A finding that has key medical relevance is that when we inhibited Pol $\theta$ function in cells lacking Brcal or Brca2, cell survival was affected," states Sfeir. D'Andrea and colleagues expanded this observation to an ovarian cancer cell line deficient in HR due to depletion of FANCD2, and mice lacking both Fancd2 and Polq (PolO). "Double knockout of the HR pathway and the PARP1-PolO MMEJ pathway was synthetic lethal in tumour cells and was embryonic lethal in our double knockout mouse model," says D’Andrea. In particular, depletion of $\mathrm{Pol} \theta$ decreased

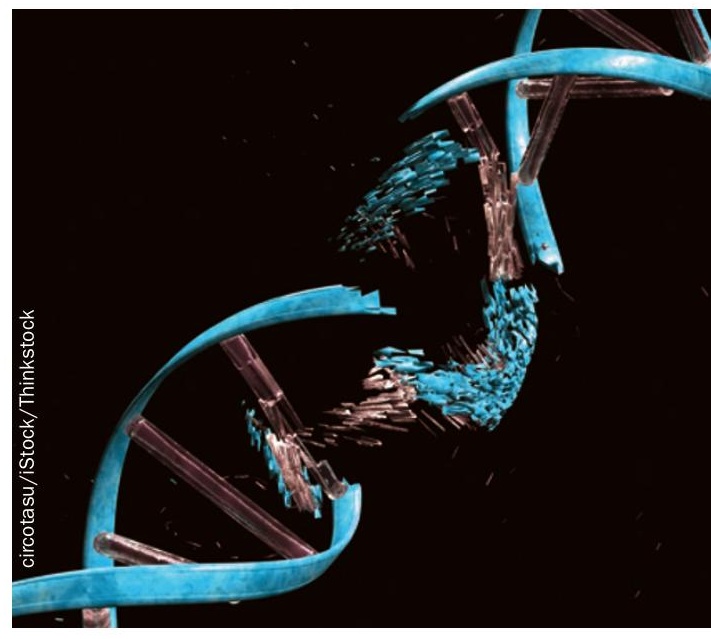

the survival of the HR-deficient ovarian cells upon PARP inhibition (PARPi), or treatment with cisplatin or mitomycin C. Furthermore, conditional knockdown of PolO in xenografts formed from these cells rendered them more sensitive to PARPi and conferred a survival advantage, compared with controls retaining Pol $\theta$ expression.

"Our study predicts that $\operatorname{Pol} \theta$ inhibitors could be used (alone or in combination with other DNA damaging therapies) to treat tumours that are deficient for $\mathrm{HR}$, including those lacking BRCA1/2," Sfeir opines. "We found that PolO ATPase activity is required for its anti-recombinase function and, therefore, we will perform a high-throughput screening assay for inhibitors of PolO ATPase activity," adds D'Andrea. "As we show that Pol $\theta$ depletion in vivo sensitizes HR-deficient ovarian tumour cells to PARPi treatment, finding small-molecule inhibitors of PolO ATPase activity will offer new potential therapeutic options for ovarian cancers or other cancers with inactivated HR," he concludes. When and why tumours opt to use the error-prone MMEJ repair, and whether this contributes to the mutation profile of tumours are important questions for future studies.

David Killock 